Chun-Jung Juan

Cheng-Yu Chen

Yi-Jui Liu

Hsiao-Wen Chung

Shy-Chy Chin

Chun-Jen Hsueh

Hsin Chu

Robert A. Zimmerman

\section{Acute putaminal necrosis and white matter demyelination in a child with subnormal copper metabolism in Wilson disease: MR imaging and spectroscopic findings}

Received: 29 July 2003

Accepted: 9 October 2004

Published online: 21 May 2005

(c) Springer-Verlag 2005

\section{C.-J. Juan · H.-W. Chung}

Department of Electrical Engineering,

College of Electrical Engineering

and Computer Sciences,

National Taiwan University,

Taipei, Taiwan

C.-J. Juan · C.-Y. Chen $(\varangle)$

H.-W. Chung - S.-C. Chin - C.-J. Hsueh

Department of Radiology,

Tri-Service General Hospital,

325, Section 2, Cheng-Kung Road,

Nei-Hu 114, Taipei, Taiwan

E-mail: sandy0928@seed.net.tw

Tel: + 886-2-87927244

Fax: + 886-2-87927245

Y.-J. Liu

Department of Automatic Control Engineering, Feng Chia University,

Taichung, Taiwan

H. Chu

Department of Neurology,

National Defense Medical Center,

Taipei, Taiwan

R. A. Zimmerman

Department of Radiology,

The Children's Hospital of Philadelphia,

Philadelphia, Pennsylvania, USA

\author{
Abstract Wilson disease (WD) that \\ manifests solely with acute and \\ severe neurological damage in the \\ absence of hepatic disease and \\ Kayser-Fleischer ring of the cornea \\ is rare and difficult to diagnose at the \\ acute setting. This report describes \\ unusual diffusion and proton \\ spectroscopic magnetic resonance \\ (MR) imaging findings in a \\ 12-year-old boy with WD who \\ presented with hemichorea and \\ subnormal copper metabolism. The \\ MR imaging findings of lactate \\ accumulation, decrease of $N$-aceryl- \\ aspartate/creatinine (NAA/Cr) ratio \\ and markedly increased apparent \\ diffusion coefficient (ADC) value of \\ the asymmetrical edematous \\ putaminal lesions in the early stage \\ were suggestive of acute necrosis \\ with anaerobic metabolism of \\ glucose leading to poor clinical \\ outcome at follow-up.
}

Keywords Wilson disease · Vasogenic edema $\cdot$ Anaerobic metabolism $\cdot$ MR imaging $\cdot$ Basal ganglia

\section{Introduction}

Neurodegenerative Wilson disease (NWD) is a genetic disease caused by mutations in a P-type ATPase; the gene is localized on chromosome 13. The defect leads to abnormal copper transport and metabolism in mitochondria and causes apoptotic and necrotic cell death due to oxidative damage after excess accumulation of intracellular copper [1]. Neurological symptoms, when present, usually follow the typical course of hepatolenticular degeneration, with clinical presentations of overwhelming hepatic and ocular copper accumulation, i.e., hepatic dysfunction and the typical Kayser-Fleischer ring of the cornea. When patients manifest solely 
with neurological symptoms, diagnosis is usually difficult, with a mean delay of 12.8 years reported [2]. Reports of the severe form of NWD in an early stage are uncommon [3].

The reported cerebral magnetic resonance (MR) imaging findings of NWD have shown T2 hyperintense lesions involving mostly the putamina and, less frequently, the thalami, brain stem, cerebellar dentate regions, and cerebral white matter $[4,5]$. Most of the conventional MR images were acquired on patients with typical hepatolenticular degeneration who had known abnormal copper metabolism and chronic hepatic failure. More recently, the application of advanced MR techniques, such as diffusion imaging and proton spectroscopy, has shed a better light on the pathogenesis of NWD by inspecting the microscopic water diffusion and cellular metabolism in vivo [6-10]. The MR imaging findings of an acute neurological form of Wilson disease (WD) have not been previously reported. We describe the MR diffusion and proton spectroscopic imaging findings of a 12-year-old boy with acute NWD in the absence of hepatic abnormality.

\section{Case report}

A previously healthy 12 -year-old child presented with a 2-month history of clumsiness, with involuntary movement of his left upper limb (hemichorea) following a 3-day-febrile upper respiratory infection. The symptoms ceased while the child was sleeping. Physical examinations were unremarkable except for the neurological examination, which showed an involuntary dystonic movement of the left upper limb and rigidity of the left shoulder, elbow and wrist joints. Slit-light examination showed no abnormal deposition in the cornea. Studies of the blood and the CSF excluded bacterial or viral infection. There was a decreased serum copper level [65.4 $\mu \mathrm{g} / \mathrm{dl}$ (normal range: $85 \mu \mathrm{g} / \mathrm{dl}-155 \mu \mathrm{g} / \mathrm{dl}$ )] and serum ceruloplasmin level $[13.6 \mathrm{mg} / \mathrm{dl}$ (normal range $22-58 \mathrm{mg} / \mathrm{dl}$ )] and normal 24-h urine copper excretion $[42.7 \mu \mathrm{g} / 24 \mathrm{~h}$ (normal range $<100 \mu \mathrm{g} / 24 \mathrm{~h}$ )]. The serum aspartate aminotransferase and the serum glutamic pyruvic transaminase were within normal limits. Chest radiographs, echocardiograms and abdominal sonograms were unremarkable.

Magnetic resonance study was performed with a 1.5-T scanner (Siemens, Vision Plus, Erlangen, Germany) using axial spin echo T1-weighted (TR/TE/excitations: 700/14/1), T2-weighted $(4000 / 99 / 2)$ and fluid attenuated inversion recovery $(9000 / 110 / 1)$ images. Postcontrast T1-weighted images were obtained following intravenous injection of gadolinium at a dose of $0.2 \mathrm{mmol} / \mathrm{kg}$. Diffusion-weighted images were acquired using an echoplanar sequence with TR of $4,251 \mathrm{~ms}$, TE of $110 \mathrm{~ms}$, a matrix size of $256 \times 256$ pixels, and slice thickness of $6 \mathrm{~mm}$ in a total of 20 slices. Diffusion values with $b=0,500$ and $1,000 \mathrm{~s} / \mathrm{mm}^{2}$ at three orthogonal gradient axes $(x, y$ and $z$ ) were applied with apparent diffusion coefficient (ADC) maps automatically generated. We calculated the ADC values semi-automatically by manually circling a region of interest of $0.3 \mathrm{~cm}^{2}$ within the lesion. In addition, a control diffusionweighted MR study was carried out on 15 age-matched (mean 9.3 years, range 5 years to 18 years) healthy individuals who underwent identical MR examinations on the same scanner. The normal mean ADC value (mean \pm standard deviation) of the putamina was $0.74 \pm 0.05 \times 10^{-3} \mathrm{~mm}^{2} / \mathrm{s}$ on the right and $0.74 \pm 0.08 \times$ $10^{-3} \mathrm{~mm}^{2} / \mathrm{s}$ on the left, ranging from $0.66 \times 10^{-3} \mathrm{~mm}^{2} / \mathrm{s}$ to $0.84 \times 10^{-3} \mathrm{~mm}^{2} / \mathrm{s}$ and $0.64 \times 10^{-3} \mathrm{~mm}^{2} / \mathrm{s}$ to $0.92 \times 10^{-3} \mathrm{~mm}^{2} / \mathrm{s}$, respectively. ${ }^{1} \mathrm{H}$-proton MR spectroscopy, using a single voxel PRESS-based technique with TR $1,500 \mathrm{~ms}$, TE $135 \mathrm{~ms}$ and a spatially localized voxel size of $3 \times 2.2 \times 2 \mathrm{~cm}^{3}$ in the right putaminal lesion and $4.2 \times 2.5 \times 2 \mathrm{~cm}^{3}$ in the unaffected occipital lobe, served as a reference. The conventional MR images showed asymmetrical basal ganglionic lesions presenting as low signal intensity on T1-weighted image (T1WI) and markedly high signal intensity on T2-weighted image (T2WI) (Fig. 1a). Moreover, there was increased signal intensity on T2WI of the occipital peri-ventricular and subcortical white matter, bilaterally, without mass effect, suggestive of demyelination. In contrast to the lesion on the left side that conformed to the boundary of the putamen, the right basal ganglionic lesion involved not only putamen but also the globus pallidus and the corona radiata, consistent with hemichorea in the left limb. The lesions showed hyperintensity on apparent coefficient maps with increased ADC values, with the right-sided lesion larger than the left (right: $1.86 \pm 0.14 \times 10^{-3} \mathrm{~mm}^{2} / \mathrm{s}$; left: $\left.1.24 \pm 0.08 \times 10^{-3} \mathrm{~mm}^{2} / \mathrm{s}\right)$ (Fig. 1b) and hypointensity on diffusion-weighted images (Fig. 1c). Following intravenous injection of gadolinium, there was no abnormal enhancement of the basal ganglionic lesion or the white matter on T1WI. Single voxel proton MR spectroscopy (Fig. 1d) with the sampling box placed in the right putaminal lesion showed a low $\mathrm{N}$-acerylaspartate/creatinine (NAA/Cr) ratio (0.98), a normal choline (Cho)/Cr ratio (0.85) and a high lactate (Lac) $/ \mathrm{Cr}$ ratio (0.55), in contrast to those of the bilateral occipital lobes (NAA/Cr ratio 1.92; no lactate accumulation). WD with acute putaminal necrosis and white matter desmyelination or demyelination was diagnosed on the basis of the clinical presentation, subnormal copper metabolic profile and MR imaging findings. The administration of copper-chelating agents, including Dpenicillamine and zinc acetate, were initiated, and a lowcopper diet was suggested.

Six months after discharge, the child was admitted again owing to the progression of the disease, with a clinical presentation of rigidity, dystonic posture and 

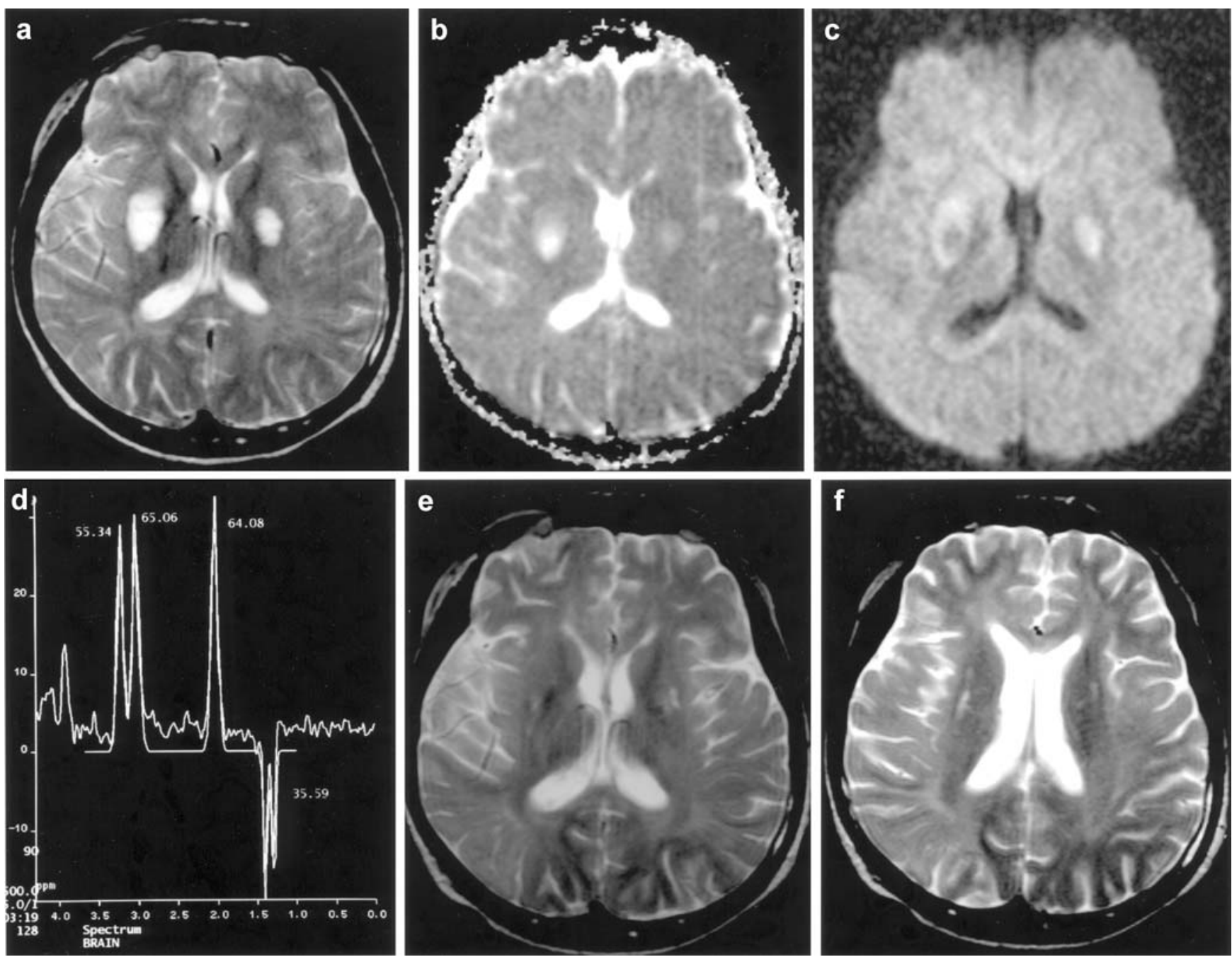

Fig. 1 A 12-year-old child with WD and subnormal copper metabolism. Initial MR study at the level of the basal ganglia shows asymmetrical putaminal hyperintense lesion on T2WI (a). The lesions are hyperintense on ADC maps (b) and hypointense on diffusion-weighted images $(b=1,000)(\mathbf{c}) .{ }^{1} \mathrm{H}$-proton MR spectroscopy shows a decreased NAA/Cr ratio, normal $\mathrm{Cho} / \mathrm{Cr}$ ratio and elevated $\mathrm{Lac} / \mathrm{Cr}$ ratio (d). A repeated MR study 6 months later shows collapse of the bilateral putamina and diffuse white matter hyperintensity over the bilateral cerebral hemispheres on T2WI $(\mathbf{e}, \mathbf{f})$

aphasia. Impaired leaning and memory had developed gradually. On this admission the laboratory study showed elevation of serum aspirate aminotransferase (88 U/1, reference value $10 \mathrm{U} / 1-34 \mathrm{U} / 1$ ) and serum glutamic pyruvic transaminase $(39 \mathrm{U} / 1$, reference value $7 \mathrm{U} / 1-33 \mathrm{U} / \mathrm{l})$. The serum copper and ceruloplasmin levels returned to normal range $(91.4 \mu \mathrm{g} / \mathrm{dl}$ and $24.6 \mathrm{mg} / \mathrm{dl}$, respectively) and the 24-h urine copper excretion was normal $(30 \mu \mathrm{g} / \mathrm{dl})$. MR images revealed collapse of the putamina bilaterally, with a few, tiny, residual, bright signal spots on T2WI (Fig. 1e). The
ADC values of the locations corresponding to the previous putaminal lesions were slightly higher on the right and normal on the left compared with the control group $\left(0.89 \pm 0.11 \times 10^{-3} \mathrm{~mm}^{2} / \mathrm{s}\right.$ on the right and $0.78 \pm 0.09 \times$ $10^{-3} \mathrm{~mm}^{2} / \mathrm{s}$ on the left). The white matter hyperintensity became more extensive on T2WI, as seen from adjacent slices, such as that shown in Fig. 1f, indicating more generalized white matter demyelination.

\section{Discussion}

Wilson disease (hepatolenticular degeneration) is an autosomal recessive disease caused by mutations in the ATPFB (copper-binding ATPase) gene located at band q14.3 of chromosome 13. The peak age at presentation is between 8 years and 16 years. The impairment of copper transport across membranes leads to accumulation of copper in the liver, brain, cornea and kidney, and causes toxicity to these organs. The damaged copper transport 
secondarily causes a low serum ceruloplasmin level. The diagnosis is not difficult when the disease presents predominantly as hepatic failure (jaundice or portal hypertension) and the pathognomonic Kayser-Fleischer ring of the cornea is present and there are low levels of serum copper and ceruloplasmin and an increase in urinary copper excretion. The diagnosis of WD can be difficult in the absence of hepatic disease and corneal Kayser-Kleischer ring with normal or mildly abnormal laboratory results. The neurological symptoms, such as indistinct speech, dysarthria, tremor, rigidity, dystonia, gait difficulty, uncoordination, difficulty with fine motor tasks, intellectual impairment or emotional disturbances, are neither specific nor sufficient for diagnosis of NWD and may be secondary to a wide spectrum of diseases.

In NWD, the lesions tend to be bilateral and often symmetrical and may involve gray and white matter. The putamen, caudate nucleus, globus pallidus, claustrum, thalamus, cortical or subcortical regions, mesencephalon, pons, vermis and dentate nucleus are all targets of injury. MR study has successfully demonstrated the brain lesions with high signal intensity on T2WI in NWD [4, 5]. However, the correlation among the T2 hyperintense lesions, the severity of copper metabolism and liver disease, and the histological changes of edema, necrosis and spongiform degeneration has not been fully investigated.

The water diffusion characteristics of NWD lesions have been reported by a few investigators; studies that included acute hepatolenticular degenerative patients have shown restriction of water diffusion of the basal ganglionic lesions, suggesting cytotoxic edema [6, 7]. On the other hand, the white matter abnormalities could also show increased water diffusion, presumably due to vasogenic edema or demyelination [6]. In our patient, the increased ADC values of basal ganglionis lesions on diffusion-weighted images suggested an evolution of neuronal necrosis after initial cytotoxic edema.

Studies from MR spectroscopy on WD generally revealed neuronal loss reflected by a decrease in the NAA/ $\mathrm{Cr}$ and $\mathrm{Cho} / \mathrm{Cr}$ ratios of the lesions [8-10]. However, none of the studies showed accumulation of lactate in the putaminal lesions. Lactate, a product of anaerobic metabolism, is not found in elevated levels in a healthy brain. It is present in patients with mitochondrial disorders such as Kearns-Sayre syndrome, mitochondrial myopathy, encephalopathy, lactic acidosis and strokelike episodes (MELAS), myoclonic epilepsy with ragged red fibers (MERRF) and Leigh disease (subacute necrotizing encephalomyopathy) [11]. It may also be observed in patients with acute ischemic cerebral infarction, cardiac arrest, hypoxia or hypoglycemic brain injury. The presence of elevated lactate represents the defect in aerobic metabolism in mitochondria and the presence of anaerobic metabolism. In WD, the gene defect (P-ATPase) affects the function of cytochrome C oxidase at the level of the mitochondria [12]. Cytochrome $\mathrm{C}$ oxidase (COX-complex IV) requires copper, to function. In the absence of functional copper, there may be COX deficiency, with resultant elevation of lactate. In our cases, the high $\mathrm{Lac} / \mathrm{Cr}$ and low NAA/Cr ratios further indicated possible anaerobic metabolism and necrosis with neuronal loss in the lesions. This was followed by eventual putaminal necrosis and atrophy, seen on the follow-up images.

In summary, NWD may present in childhood with neurological signs due to fulminant putaminal necrosis without hepatic dysfunction or corneal copper deposition, as in our case. The presence of lactate accumulation in the edematous putaminal lesion suggests failure of aerobic respiration of the brain cells and the start of apoptosis. The diagnosis of NWD requires a high index of clinical suspicion and MR imaging findings of bilateral putaminal necrosis at an acute stage.

\section{References}

1. Beal MF (2000) Neurodegenerative disease. Trends Neurosci 23:298-304

2. Walshe JM, Yealland M (1992) Wilson's disease: the problem of delayed diagnosis. J Neurol Neurosurg Psychiatry 55:692-696

3. Angius A, Dessi V, Lovicu M, De Virgiliis S, Pirastu M, Cao A (1998) Early and severe neurological features in a Wilson disease patient compound heterozygous for two frameshift mutations. Eur J Pediatr 157:128-129
4. King AD, Walshe JM, Kendall BE, et al (1996) Cranial MR imaging in Wilson's disease. AJR Am J Roentgenol 167:1579-1584

5. van Wassenaer-van Hall $\mathrm{HN}$, van den Heuvel AG, Jansen GH, Hoogenraad TU, Mali WP (1995) Cranial MR in Wilson disease: abnormal white matter in extrapyramidal and pyramidal tracts AJNR Am J Neuroradiol 16:2021-2027

6. Sener RN (2003) Diffusion MRI findings in Wilson's disease. Comput Med Imaging Graph 27:17-21
7. Sener RN (2003) Diffusion MR imaging changes associated with Wilson disease. AJNR Am J Neuroradiol 24:965-967

8. Jayasundar R, Sahani AK, Gaikwad S, Singh S, Behari M (2002) Proton MR spectroscopy of basal ganglia in Wilson's disease: case report and review of literature. Magn Reson Imaging 20:131-135 
9. van der Heuvel AG, van der Grond J, van Rooij LG, van Wassenaer-van Hall HN, Hoogenraad TU, Mali WP (1997) Differentiation between portal-systemic encephalopathy and neurodegenerative disorders in patients with Wilson disease: H-1 MR spectroscopy. Radiology 203:539-543
10. Alanen A, Komu M, Penttinen M, Leino R (1999) Magnetic resonance imaging and proton MR spectroscopy in Wilson's disease. Br J Radiol 72:749756

11. Nusbaum AO, Fung KM, Atlas SW (2002) White matter diseases and inherited metabolic disorders. In: Altas SW (ed) Magnetic resonance imaging of the brain and spine. Lippincott Williams\& Wilkins, Philadelphia, pp 521563
12. Sternlieb I (1968) Mitochondrial and fatty changes in hepatocytes of patients with Wilson's disease. Gastroenterology 55:354-367 